\title{
Local saturation of a positive linear convolution operator
}

Hee Sun Jung ${ }^{1 *}$ and Ryozi Sakai ${ }^{2}$

\section{${ }^{*}$ Correspondence:}

hsun90@skku.edu

${ }^{1}$ Department of Mathematics

Education, Sungkyunkwan

University, Seoul, 110-745, Republic of Korea

Full list of author information is available at the end of the article

\section{Abstract}

Let $\left\{H_{n}(t)\right\}$ be a sequence of non-negative, even, and continuous functions on $\mathbb{R}$. In this paper, we consider a convolution operator $J_{n}(f ; x)=\int_{0}^{\infty} f(t) H_{n}(t-x) d t, f \in L_{p}\left(\mathbb{R}^{+}\right)$, and then investigate the local saturation of $J_{n}(f ; x)$.

MSC: $44 \mathrm{~A} 35$

Keywords: convolution operator; local saturation

\section{Introduction and theorems}

Through this paper we let $\left\{H_{n}(t)\right\}, n=1,2, \ldots$, be a sequence of non-negative, even, and continuous functions on $\mathbb{R}:=(-\infty, \infty)$, and there exist $M, N>0$, and $T>0$ such that $\left\{H_{n}(t)\right\}$ satisfied uniformly

$$
\begin{aligned}
& \sup _{|t| \geq T} H_{n}(t) \leq M, \quad n \geq N, \\
& \int_{-\infty}^{\infty} H_{n}(t) d t=1, \quad n=1,2, \ldots, \\
& \int_{-\infty}^{\infty} t^{2} H_{n}(t) d t=\mu_{n} \rightarrow 0 \quad \text { as } n \rightarrow \infty
\end{aligned}
$$

and there exist two positive constants $\alpha, \beta$ with $2 \beta \geq \alpha>3$ such that uniformly for $n$,

$$
\int_{-\infty}^{\infty}|t|^{\alpha} H_{n}(t) d t \leq C \mu_{n}^{\beta}
$$

As an example for $H_{n}(t)$ we give the following:

Let

$$
H_{n}(t)=\frac{n}{\sqrt{\pi}} e^{-(n t)^{2}} .
$$

Then it satisfies (1.2),

$$
\mu_{n}=\frac{1}{n^{2}} \rightarrow 0 \quad \text { as } n \rightarrow \infty \quad \text { and } \quad \int_{-\infty}^{\infty} t^{4} H_{n}(t) d t=\frac{3}{4} \frac{1}{n^{4}} .
$$

O2014 Jung and Sakai; licensee Springer. This is an Open Access article distributed under the terms of the Creative Commons Attribution License (http://creativecommons.org/licenses/by/2.0), which permits unrestricted use, distribution, and reproduction in any medium, provided the original work is properly cited. 
Let us denote $\mathbb{R}^{+}:=[0, \infty)$. In what follows we assume that $H_{n}(t)$ satisfies the conditions (1.1)-(1.4). Using $H_{n}$, we define the convolution operators for $f \in L_{p}\left(\mathbb{R}^{+}\right)$,

$$
J_{n}(f ; x):=\int_{0}^{\infty} f(t) H_{n}(t-x) d t, \quad n=1,2, \ldots
$$

Swetits and Wood [1] studied the operators on a finite interval $[0, r]$;

$$
K_{n}(f ; x):=\int_{0}^{r} f(t) H_{n}^{*}(t-x) d t, \quad f \in L_{p}([0, r]), n=1,2, \ldots,
$$

where $H_{n}^{*}$ is defined on $[-r, r]$ as $H_{n}$, and then they gave a local saturation theorem. Furthermore, there is a rich bibliography concerning the convergence of positive linear operators on $[0, \infty)$ (e.g. see $[2-4]$ and the references cited therein).

In this paper we extend [1] to the infinite interval $\mathbb{R}^{+}$. Then we use a similar methods as [1]. For $1<p<\infty$ and $c \geq 0$, we define $L_{p}^{2}([c, \infty))$ as the space of those functions $f$ such that $f \in L_{p}\left(\mathbb{R}^{+}\right)$and $f^{\prime}$ is a locally absolutely continuous function on $[c, \infty)$, with $f^{\prime} \in L_{p}([c, \infty))$ and $f^{\prime \prime} \in L_{p}([c, \infty))$. Let $C_{c}^{2}\left(\mathbb{R}^{+}\right)$be the space of continuous, compactly supported and continuously second differentiable functions on $\mathbb{R}^{+}$. Furthermore, the total variation of a real-valued function $f$ defined on an interval $[a, b] \subset \mathbb{R}$ is the quantity

$$
V_{a}^{b}(f)=\sup _{P \in \mathcal{P}} \sum_{i=0}^{n_{P}-1}\left|f\left(x_{i+1}\right)-f\left(x_{i}\right)\right|
$$

Here the supremum is taken over the set $\mathcal{P}$ of all partitions $P=\left\{x_{0}, x_{1}, \ldots, x_{n_{P}}\right\}$ of the interval considered. If $F:[b, \infty) \rightarrow \mathbb{R}$ and $x \in[b, \infty)$, we define

$$
T_{F}(x)=\sup \left\{\sum_{1}^{n}\left|F\left(x_{j}\right)-F\left(x_{j-1}\right)\right|: b=x_{0}<x_{1}<\cdots<x_{n}=x, n \in \mathbb{N}\right\} .
$$

If $T_{F}(\infty):=\lim _{x \rightarrow \infty} T_{F}(x)$ is finite, we say that $F$ is of bounded variation on $[b, \infty)$, and $T_{F}(\infty)$ is called the total variation of $F$ on $[b, \infty)$. We define $\mathrm{BV}[b, \infty)$ to be the set of all functions on $[b, \infty)$ whose total variation on $[b, \infty)$ is finite.

Then we first give the pointwise convergence theorem.

Theorem 1.1 (cf. [5]) Let $f \in C_{c}^{2}\left(\mathbb{R}^{+}\right)$, and let $x \in[a, \infty), a>0$. Then we have a pointwise convergence as follows:

$$
\lim _{n \rightarrow \infty} \frac{1}{\mu_{n}}\left(J_{n}(f ; x)-f(x)\right)=\frac{1}{2} f^{\prime \prime}(x)
$$

Equation (1.6) holds uniformly on $[a, \infty)$.

Then the following is a direct convergence theorem.

Theorem 1.2 Let $0<b<a<\infty$.

(i) If $1<p<\infty$, then we have for $f \in L_{p}^{2}([b, \infty))$

$$
\left\|J_{n}(f ; x)-f(x)\right\|_{L_{p}([a, \infty))}=O\left(\mu_{n}\right) \quad \text { as } n \rightarrow \infty .
$$


(ii) If $p=1$, then we have for $f^{\prime} \in \mathrm{BV}([b, \infty))$ with $f \in L_{1}([0, \infty))$

$$
\left\|J_{n}(f ; x)-f(x)\right\|_{L_{1}([a, \infty))}=O\left(\mu_{n}\right) \quad \text { as } n \rightarrow \infty .
$$

(iii) Let $1 \leq p<\infty$ and $f$ be linear on $[b, \infty)$. Then we have

$$
\left\|J_{n}(f ; x)-f(x)\right\|_{L_{p}([a, \infty))}=o\left(\mu_{n}\right) \quad \text { as } n \rightarrow \infty .
$$

Finally, we give an inverse theorem as follows.

Theorem 1.3 Let $0<b<a<\infty$. Let $f \in L_{p}\left(\mathbb{R}^{+}\right)$and $f^{\prime} \in L_{p}\left(\mathbb{R}^{+}\right)$.

(i) For $1<p<\infty$, the condition (1.7) implies $f \in L_{p}^{2}([a, \infty))$.

(ii) For $p=1$, the condition (1.8) implies $f^{\prime} \in \mathrm{BV}([a, \infty))$.

(iii) For $1 \leq p<\infty$, the condition (1.9) implies that $f$ is linear on $[a, \infty)$.

This paper is organized as follows. In Section 2, we will give some fundamental lemmas in order to prove the main results and we will prove the results in Section 3.

\section{Fundamental lemmas}

Throughout this paper $C, C_{1}, C_{2}, \ldots$ denote positive constants independent of $n, x, t$ or function $f(x)$. The same symbol does not necessarily denote the same constant in different occurrences.

To prove the theorems we need some lemmas.

Lemma 2.1 Let $\delta>0$ and $\ell=0,1,2$. Then for $2 \beta \geq \alpha>3$ defined in (1.4)

$$
\int_{|u| \geq \delta}|u|^{\ell} H_{n}(u) d u \leq C \frac{\mu_{n}^{\beta}}{\delta^{\alpha-\ell}}
$$

Proof Let $\ell=0,1,2$. Then we have from (1.4)

$$
\begin{aligned}
\int_{|u| \geq \delta}|u|^{\ell} H_{n}(u) d u & =\delta^{\ell} \int_{|u| \geq \delta}\left(\frac{|u|}{\delta}\right)^{\ell} H_{n}(u) d u \\
& \leq \delta^{\ell} \int_{|u| \geq \delta}\left(\frac{|u|}{\delta}\right)^{\alpha} H_{n}(u) d u \leq \delta^{\ell-\alpha} \int_{|u| \geq \delta}|u|^{\alpha} H_{n}(u) d u \\
& =\delta^{\ell-\alpha} \int_{-\infty}^{\infty}|u|^{\alpha} H_{n}(u) d u \leq C \frac{\mu_{n}^{\beta}}{\delta^{\alpha-\ell}} .
\end{aligned}
$$

Lemma 2.2 Let $e_{0}(x):=1, e_{1}(x):=x$ and let $\delta$ be a positive constant. Then for $n=1,2, \ldots$, we have

$$
\left|J_{n}((\cdot-x) ; x)\right| \leq C \frac{\mu_{n}^{\beta}}{\delta^{\alpha-1}}, \quad 0<\delta \leq x
$$

and

$$
\left|J_{n}\left((\cdot-x)^{2} ; x\right)\right| \leq \mu_{n}, \quad x \geq 0
$$


Jung and Sakai Journal of Inequalities and Applications 2014, 2014:329

Page 4 of 16

Moreover, we have for $0<\delta \leq x$

$$
\left|J_{n}\left(e_{0} ; x\right)-e_{0}\right| \leq C \frac{\mu_{n}^{\beta}}{\delta^{\alpha}} \quad \text { and } \quad\left|J_{n}\left(e_{1} ; x\right)-e_{1}\right| \leq C \frac{\mu_{n}^{\beta}}{\delta^{\alpha-1}}
$$

Here, $\alpha$ and $\beta$ are defined in (1.4).

Proof For $0<\delta \leq x$, we have by $(2.1)$

$$
\begin{aligned}
\left|J_{n}((\cdot-x) ; x)\right| & =\left|\int_{0}^{\infty}(t-x) H_{n}(t-x) d t\right|=\left|\int_{-x}^{\infty} y H_{n}(y) d y\right| \\
& =\left|\int_{x}^{\infty} y H_{n}(y) d y\right| \quad \because H_{n}(t) \text { is even } \\
& \leq \int_{y \geq \delta} y H_{n}(y) d y \leq C \frac{\mu_{n}^{\beta}}{\delta^{\alpha-1}} .
\end{aligned}
$$

For (2.3), we have from (1.3)

$$
J_{n}\left((\cdot-x)^{2} ; x\right)=\int_{0}^{\infty}(t-x)^{2} H_{n}(t-x) d t=\int_{-x}^{\infty} y^{2} H_{n}(y) d y \leq \mu_{n}
$$

Since we know for $0<\delta \leq x$

$$
J_{n}\left(e_{0} ; x\right)=\int_{0}^{\infty} H_{n}(t-x) d t=\int_{-x}^{\infty} H_{n}(y) d y=1-\int_{-\infty}^{-x} H_{n}(y) d y=1-\int_{x}^{\infty} H_{n}(y) d y
$$

and from (2.1)

$$
\left|\int_{x}^{\infty} H_{n}(y) d y\right| \leq C \frac{\mu_{n}^{\beta}}{\delta^{\alpha}}
$$

we have for $0<\delta \leq x$,

$$
\left|J_{n}\left(e_{0} ; x\right)-e_{0}\right| \leq C \frac{\mu_{n}^{\beta}}{\delta^{\alpha}}
$$

Next, we give an estimate for $e_{1}$. Since

$$
\begin{aligned}
J_{n}\left(e_{1} ; x\right) & =\int_{0}^{\infty} t H_{n}(t-x) d t=\int_{-x}^{\infty}(x+y) H_{n}(y) d y \\
& =x\left(1-\int_{-\infty}^{-x} H_{n}(y) d y\right)+\int_{x}^{\infty} y H_{n}(y) d y \\
& =x-x \int_{x}^{\infty} H_{n}(y) d y+\int_{x}^{\infty} y H_{n}(y) d y
\end{aligned}
$$

and by (2.1), for $0<\delta \leq x$,

$$
\left|x \int_{x}^{\infty} H_{n}(y) d y\right|+\left|\int_{x}^{\infty} y H_{n}(y) d y\right| \leq 2 \int_{x}^{\infty} y H_{n}(y) d y \leq C \frac{\mu_{n}^{\beta}}{\delta^{\alpha-1}}
$$


we have for $0<\delta \leq x$,

$$
\left|J_{n}\left(e_{1} ; x\right)-e_{1}\right| \leq C \frac{\mu_{n}^{\beta}}{\delta^{\alpha-1}} .
$$

Let $b>0$ and then we define

$$
\chi([0, b] ; t):= \begin{cases}1, & t \in[0, b], \\ 0, & t \notin[0, b] .\end{cases}
$$

Lemma 2.3 Let $0<b<a, \delta:=a-b$ and let $1 \leq p<\infty$. Iff $\in L_{p}\left(\mathbb{R}^{+}\right)$, then

$$
\left\|J_{n}(\chi([0, b]) f)\right\|_{L_{p}[a, \infty)} \leq C \frac{\mu_{n}^{\beta}}{\delta^{\alpha}}\|f\|_{L_{p}\left(\mathbb{R}^{+}\right)} .
$$

Proof Let $p=1$. Then we have

$$
\begin{aligned}
\left\|J_{n}(\chi([0, b]) f)\right\|_{L_{1}([a, \infty))} & =\int_{a}^{\infty}\left|\int_{0}^{\infty} \chi([0, b] ; t) f(t) H_{n}(t-x) d t\right| d x \\
& \leq \int_{a}^{\infty} \int_{0}^{b}|f(t)| H_{n}(t-x) d t d x \\
& \leq \int_{0}^{b}|f(t)| \int_{a}^{\infty} H_{n}(t-x) d x d t \\
& \leq\left(\sup _{t \in[0, b]} \int_{a}^{\infty} H_{n}(t-x) d x\right)\|f\|_{L_{1}\left(\mathbb{R}^{+}\right)} .
\end{aligned}
$$

For $t \in[0, b]$ and $x \geq a$, we see $|t-x| \geq a-b=: \delta>0$. From (1.4), we have the following:

$$
\begin{aligned}
\sup _{t \in[0, b]} \int_{a}^{\infty} H_{n}(t-x) d x & \leq \sup _{t \in[0, b]} \int_{a}^{\infty}\left(\frac{|t-x|}{\delta}\right)^{\alpha} H_{n}(t-x) d x \\
& \leq \frac{1}{\delta^{\alpha}} \int_{-\infty}^{\infty}|t|^{\alpha} H_{n}(t) d t \leq C \frac{\mu_{n}^{\beta}}{\delta^{\alpha}} .
\end{aligned}
$$

Hence we have

$$
\left\|J_{n}(\chi([0, b]) f)\right\|_{L_{1}[a, \infty)} \leq C \frac{\mu_{n}^{\beta}}{\delta^{\alpha}}\|f\|_{L_{1}\left(\mathbb{R}^{+}\right)} .
$$

Let $1<p<\infty, 0<b<a \leq x$ and $p+q=p q$. By Hölder's inequality,

$$
\begin{aligned}
\left|J_{n}(\chi([0, b]) f, x)\right|= & \left|\int_{0}^{\infty} \chi([0, b] ; t) f(t) H_{n}(t-x) d t\right| \\
\leq & C\left(\int_{0}^{\infty} \chi([0, b] ; t) H_{n}(t-x) d t\right)^{1 / q} \\
& \times\left(\int_{0}^{\infty} \chi([0, b] ; t)|f(t)|^{p} H_{n}(t-x) d t\right)^{1 / p} .
\end{aligned}
$$


Here, from (2.1) we have

$$
\begin{aligned}
\int_{0}^{\infty} \chi([0, b] ; t) H_{n}(t-x) d t & =\int_{0}^{b} H_{n}(t-x) d t=\int_{x-b}^{x} H_{n}(y) d y \\
& \leq \int_{\delta}^{\infty} H_{n}(y) d y \leq C \frac{\mu_{n}^{\beta}}{\delta^{\alpha}} .
\end{aligned}
$$

Hence,

$$
\begin{aligned}
& \left\|J_{n}(\chi([0, b]) f)\right\|_{L_{p}[a, \infty)} \\
& \quad \leq C\left(\frac{\mu_{n}^{\beta}}{\delta^{\alpha}}\right)^{1 / q}\left(\int_{a}^{\infty} \int_{0}^{\infty} \chi([0, b] ; t)|f(t)|^{p} H_{n}(t-x) d t d x\right)^{1 / p} .
\end{aligned}
$$

Also, we see by (2.6)

$$
\begin{aligned}
& \int_{a}^{\infty} \int_{0}^{\infty} \chi([0, b] ; t)|f(t)|^{p} H_{n}(t-x) d t d x \\
& \quad \leq \int_{0}^{\infty} \int_{a}^{\infty} \chi([0, b] ; t)|f(t)|^{p} H_{n}(t-x) d x d t \\
& \quad \leq \sup _{t \in[0, b]} \int_{a}^{\infty} H_{n}(t-x) d x \int_{0}^{\infty}|f(t)|^{p} d t \leq C\|f\|_{L_{p}\left(\mathbb{R}^{+}\right)}^{p} \frac{\mu_{n}^{\beta}}{\delta^{\alpha}} .
\end{aligned}
$$

Thus, by (2.8) we conclude.

\section{Proof of theorems}

Proof of Theorem 1.1 For $x \in[a, \infty)$ and $t \geq 0$, we set

$$
f(t)=f(x)+f^{\prime}(x)(t-x)+\frac{f^{\prime \prime}(\eta)}{2}(t-x)^{2}, \quad x \lessgtr \eta \lessgtr t .
$$

Then we see

$$
\begin{aligned}
J_{n}(f ; x) & -f(x)-\frac{1}{2} f^{\prime \prime}(x) \mu_{n} \\
= & f(x)\left(J_{n}\left(e_{0} ; x\right)-e_{0}\right)+f^{\prime}(x) \int_{0}^{\infty}(t-x) H_{n}(t-x) d t \\
& +\frac{1}{2}\left[\int_{0}^{\infty} f^{\prime \prime}(\eta)(t-x)^{2} H_{n}(t-x) d t-f^{\prime \prime}(x) \mu_{n}\right], \quad x \lessgtr \eta \lessgtr t \\
:= & J_{1}+J_{2}+J_{3} .
\end{aligned}
$$

For $J_{1}$, we have from (2.4)

$$
\left|J_{1}\right|=|f(x)| O\left(\frac{\mu_{n}^{\beta}}{a^{\alpha}}\right)
$$

and for $J_{2}$, we have from (2.2)

$$
\left|J_{2}\right|=\left|f^{\prime}(x) J_{n}((\cdot-x) ; x)\right|=\left|f^{\prime}(x)\right| O\left(\frac{\mu_{n}^{\beta}}{a^{\alpha-1}}\right) .
$$


Now, we will estimate $J_{3}$. We have

$$
\begin{aligned}
& \int_{0}^{\infty} f^{\prime \prime}(\eta)(t-x)^{2} H_{n}(t-x) d t-f^{\prime \prime}(x) \mu_{n} \\
& \quad=\int_{0}^{\infty}\left(f^{\prime \prime}(\eta)-f^{\prime \prime}(x)\right)(t-x)^{2} H_{n}(t-x) d t-f^{\prime \prime}(x) \int_{-\infty}^{0}(t-x)^{2} H_{n}(t-x) d t .
\end{aligned}
$$

For the second term, we have by (2.1)

$$
\left|f^{\prime \prime}(x) \int_{-\infty}^{0}(t-x)^{2} H_{n}(t-x) d t\right| \leq\left|f^{\prime \prime}(x)\right| \int_{a}^{\infty} u^{2} H_{n}(u) d u \leq C\left|f^{\prime \prime}(x)\right| \frac{\mu_{n}^{\beta}}{a^{\alpha-2}} .
$$

For a given $\varepsilon>0$, there exists a positive constant $\delta_{1}>0$ (depending only on $\varepsilon$ ) such that for $|x-\eta|<\delta_{1}$

$$
\left|f^{\prime \prime}(x)-f^{\prime \prime}(\eta)\right|<\varepsilon
$$

For the first term, we have using (1.3)

$$
\begin{aligned}
& \left|\int_{|x-t| \leq \delta_{1}}\left(f^{\prime \prime}(\eta)-f^{\prime \prime}(x)\right)(t-x)^{2} H_{n}(t-x) d t\right| \\
& \quad \leq \varepsilon \int_{|u| \leq \delta_{1}} u^{2} H_{n}(u) d u \leq \varepsilon \mu_{n},
\end{aligned}
$$

and by (2.1)

$$
\begin{aligned}
& \left|\int_{|x-t| \geq \delta_{1}}\left(f^{\prime \prime}(\eta)-f^{\prime \prime}(x)\right)(t-x)^{2} H_{n}(t-x) d t\right| \\
& \quad \leq 2\left\|f^{\prime \prime}\right\|_{L_{\infty}(\mathbb{R})} \int_{|u| \geq \delta_{1}} u^{2} H_{n}(u) d u \leq\left\|f^{\prime \prime}\right\|_{L_{\infty}(\mathbb{R})} O\left(\frac{\mu_{n}^{\beta}}{\delta_{1}^{\alpha-2}}\right) .
\end{aligned}
$$

Then we have for some positive constants $C_{1}, C_{2}$, and $C_{3}$

$$
\begin{aligned}
\left|J_{n}(f ; x)-f(x)-\frac{1}{2} f^{\prime \prime}(x) \mu_{n}\right| \leq & C_{1}|f(x)| \frac{\mu_{n}^{\beta}}{a^{\alpha}}+C_{2}\left|f^{\prime}(x)\right| \frac{\mu_{n}^{\beta}}{a^{\alpha-1}} \\
& +\varepsilon \mu_{n}+C_{3}\left\|f^{\prime \prime}\right\|_{L_{\infty}(\mathbb{R})} \frac{\mu_{n}^{\beta}}{\delta_{1}^{\alpha-2}} .
\end{aligned}
$$

Therefore, we have for an arbitrary $\varepsilon>0$

$$
\lim _{n \rightarrow \infty} \frac{1}{\mu_{n}}\left|J_{n}(f ; x)-f(x)-\frac{1}{2} f^{\prime \prime}(x) \mu_{n}\right| \leq \varepsilon
$$

Thus, (1.6) is proved. Moreover, noting (3.1), we see that (1.6) holds uniformly on $[a, \infty)$.

Proof of Theorem 1.2 Let $\delta:=a-b>0$. (i) We start under the condition $1 \leq p<\infty$ for the convenience of considering (ii). On the way of the proof we switch over to the assumption 
with $1<p<\infty$. Let $1<p<\infty$ and let $\chi:=\chi([0, b])$ and let $\chi_{1}(t)=1-\chi(t)$. Since

$$
f(t)=(\chi f)(t)+\left(\chi_{1} f\right)(t), \quad f(x)=\left(\chi_{1} f\right)(x), \quad x \in[a, \infty)
$$

we have

$$
\left\|J_{n}(f)-f\right\|_{L_{p}([a, \infty))} \leq\left\|J_{n}(\chi f)\right\|_{L_{p}([a, \infty))}+\left\|J_{n}\left(\chi_{1} f\right)-\chi_{1} f\right\|_{L_{p}([a, \infty))}=: K_{1}+K_{2} .
$$

By Lemma 2.3,

$$
K_{1}=\left\|J_{n}(\chi f)\right\|_{L_{p}([a, \infty))} \leq C\|f\|_{L_{p}\left(\mathbb{R}^{+}\right)} \frac{\mu_{n}^{\beta}}{\delta^{\alpha}} .
$$

We estimate $K_{2}$. Let $f \in L_{p}^{2}([a, \infty))$. Then

$$
\begin{aligned}
J:= & \left\|J_{n}\left(\left(\chi_{1} f\right)(t)-\left(\chi_{1} f\right)(x), x\right)\right\|_{L_{p}([a, \infty))} \\
= & \left(\int_{a}^{\infty}\left|\int_{0}^{\infty}\left(\chi_{1}(t) f(t)-f(x)\right) H_{n}(t-x) d t\right|^{p} d x\right)^{1 / p} \\
\leq & \left(\int_{a}^{\infty}\left|\left(\int_{0}^{b}+\int_{b}^{\infty}\right)\left(\chi_{1}(t) f(t)-f(x)\right) H_{n}(t-x) d t\right|^{p} d x\right)^{1 / p} \\
\leq & \left(\int_{a}^{\infty}|f(x)|^{p}\left|\int_{0}^{b} H_{n}(t-x) d t\right|^{p} d x\right)^{1 / p} \\
& +\left(\int_{a}^{\infty}\left|\int_{b}^{\infty}(f(t)-f(x)) H_{n}(t-x) d t\right|^{p} d x\right)^{1 / p} \\
=: & I_{1}+I_{2} .
\end{aligned}
$$

Here, for the first term, using

$$
\int_{0}^{b} H_{n}(t-x) d t \leq C \frac{\mu_{n}^{\beta}}{\delta^{\alpha}}
$$

which is shown in (2.7), we have

$$
I_{1}=\left(\int_{a}^{\infty}|f(x)|^{p}\left|\int_{0}^{b} H_{n}(t-x) d t\right|^{p} d x\right)^{1 / p} \leq C\|f\|_{L_{p}([a, \infty))} \frac{\mu_{n}^{\beta}}{\delta^{\alpha}} .
$$

From this we suppose $1<p<\infty$. By

$$
f(t)-f(x)=f^{\prime}(x)(t-x)+\int_{x}^{t}(t-u) f^{\prime \prime}(u) d u
$$

we have

$$
\begin{aligned}
I_{2} & =\left(\int_{a}^{\infty}\left|\int_{b}^{\infty}(f(t)-f(x)) H_{n}(t-x) d t\right|^{p} d x\right)^{1 / p} \\
& \leq\left(\int_{a}^{\infty}\left|\int_{b}^{\infty} f^{\prime}(x)(t-x) H_{n}(t-x) d t\right|^{p} d x\right)^{1 / p}
\end{aligned}
$$




$$
\begin{aligned}
& +\left(\int_{a}^{\infty}\left|\int_{b}^{\infty} \int_{x}^{t}(t-u) f^{\prime \prime}(u) d u H_{n}(t-x) d t\right|^{p} d x\right)^{1 / p} \\
= & =I_{2,1}+I_{2,2} .
\end{aligned}
$$

Here we note that $y H_{n}(y)$ is an odd function. Then we have by $(2.1)$

$$
\begin{aligned}
\left|\int_{b}^{\infty}(t-x) H_{n}(t-x) d t\right| & =\left|\int_{b-x}^{\infty} y H_{n}(y) d y\right|=\int_{x-b}^{\infty} y H_{n}(y) d y \\
& \leq \int_{y \geq \delta} y H_{n}(y) d y \leq C \frac{\mu_{n}^{\beta}}{\delta^{\alpha-1}}
\end{aligned}
$$

and the first term $I_{2,1}$ is estimated as

$$
\begin{aligned}
I_{2,1} & =\left(\int_{a}^{\infty}\left(\left|f^{\prime}(x)\right|\left|\int_{b}^{\infty}(t-x) H_{n}(t-x) d t\right|\right)^{p} d x\right)^{1 / p} \\
& \leq C \frac{\mu_{n}^{\beta}}{\delta^{\alpha-1}}\left(\int_{a}^{\infty}\left|f^{\prime}(x)\right|^{p} d x\right)^{1 / p} \leq C\left\|f^{\prime}\right\|_{L_{p}([a, \infty))} \frac{\mu_{n}^{\beta}}{\delta^{\alpha-1}} .
\end{aligned}
$$

Now we set

$$
\theta\left(f^{\prime \prime}, x\right):=\sup _{b \leq t, t \neq x} \frac{1}{t-x} \int_{x}^{t}\left|f^{\prime \prime}(u)\right| d u, \quad a \leq x
$$

and denote the Hardy-Littlewood majorant of $f^{\prime \prime}$ at $x$. Since $f^{\prime \prime} \in L_{p}([b, \infty))$ and $1<p<\infty$, we have

$$
\left\|\theta\left(f^{\prime \prime}, x\right)\right\|_{L_{p}([a, \infty))} \leq A_{p}\left\|f^{\prime \prime}\right\|_{L_{p}([b, \infty))},
$$

where $A_{p}>0$ depend only on $p$ [6, Theorem $\left.1, \mathrm{p} .201\right]$. Since

$$
\left|\int_{x}^{t}(t-u) f^{\prime \prime}(u) d u\right| \leq \pm \int_{x}^{t}|t-u|\left|f^{\prime \prime}(u)\right| d u \leq(t-x)^{2} \frac{1}{t-x} \int_{x}^{t}\left|f^{\prime \prime}(u)\right| d u
$$

where $\pm \int_{x}^{t} d u \geq 0$, we have by (3.7) and (1.3)

$$
\begin{aligned}
I_{2,2} & \leq\left(\int_{a}^{\infty}\left|\int_{b}^{\infty}\left((t-x)^{2} \frac{1}{t-x} \int_{x}^{t}\left|f^{\prime \prime}(u)\right| d u\right) H_{n}(t-x) d t\right|^{p} d x\right)^{1 / p} \\
& \leq\left(\int_{a}^{\infty}\left|\theta\left(f^{\prime \prime} ; x\right)\right|^{p}\left|\int_{b}^{\infty}(t-x)^{2} H_{n}(t-x) d t\right|^{p} d x\right)^{1 / p} \\
& \leq \int_{-\infty}^{\infty} u^{2} H_{n}(u) d u\left(\int_{a}^{\infty}\left|\theta\left(f^{\prime \prime} ; x\right)\right|^{p} d x\right)^{1 / p} \\
& \leq A_{p} \mu_{n}\left\|f^{\prime \prime}\right\|_{L_{p}([b, \infty))} .
\end{aligned}
$$

Hence, from (3.6) and (3.8), we have for some positive constant $C>0$

$$
I_{2} \leq I_{2,1}+I_{2,2} \leq C\left(\left\|f^{\prime}\right\|_{L_{p}([a, \infty))}+\left\|f^{\prime \prime}\right\|_{L_{p}([b, \infty))}\right) \mu_{n} .
$$


So, from (3.5) and (3.9),

$$
J \leq I_{1}+I_{2} \leq C\left(\|f\|_{L_{p}\left(\mathbb{R}^{+}\right)}+\left\|f^{\prime}\right\|_{L_{p}([a, \infty))}+\left\|f^{\prime \prime}\right\|_{L_{p}([b, \infty))}\right) \mu_{n} .
$$

Now, we see by (3.10) and (2.4)

$$
\begin{aligned}
K_{2}= & \left\|J_{n}\left(\chi_{1} f\right)-\left(\chi_{1} f\right)\right\|_{L_{p}([a, \infty))} \\
\leq & \left\|J_{n}\left(\left(\chi_{1} f\right)(t)-\left(\chi_{1} f\right)(x), x\right)\right\|_{L_{p}([a, \infty))}+\left\|\left(\chi_{1} f\right)(x)\left(J_{n}\left(e_{0}, x\right)-e_{0}\right)\right\|_{L_{p}([a, \infty))} \\
= & J+\left\|\left(\chi_{1} f\right)(x)\left(J_{n}\left(e_{0}, x\right)-e_{0}\right)\right\|_{L_{p}([a, \infty))} \\
\leq & C\left(\|f\|_{L_{p}\left(\mathbb{R}^{+}\right)}+\left\|f^{\prime}\right\|_{L_{p}([a, \infty))}+\left\|f^{\prime \prime}\right\|_{L_{p}([b, \infty))}\right) \mu_{n} \\
& +\left\|J_{n}\left(e_{0}, x\right)-e_{0}\right\|_{L_{\infty}([a, \infty))}\left\|\chi_{1} f\right\|_{L_{p}([a, \infty))} \\
\leq & C_{1}\left(\|f\|_{L_{p}\left(\mathbb{R}^{+}\right)}+\left\|f^{\prime}\right\|_{L_{p}([a, \infty))}+\left\|f^{\prime \prime}\right\|_{L_{p}([b, \infty))}\right) \mu_{n}=O\left(\mu_{n}\right) .
\end{aligned}
$$

Consequently, with (3.2) and (3.3) we conclude (i).

(ii) Let $p=1$ and $f^{\prime} \in \operatorname{BV}[b, \infty)$. Then we have for $x, t \in[b, \infty)$,

$$
f(t)-f(x)=f^{\prime}(x)(t-x)+\int_{x}^{t}(t-u) d f^{\prime}(u) .
$$

On the proof of (i) we recall the part which we assumed as $p=1$. From (3.3) and (3.5) we may only estimate

$$
I_{2}^{\prime}:=\int_{a}^{\infty}\left|\int_{b}^{\infty}(f(t)-f(x)) H_{n}(t-x) d t d x\right|
$$

(see (3.4)). By (3.11) we see

$$
\begin{aligned}
I_{2}^{\prime} \leq & \left|\int_{a}^{\infty} \int_{b}^{\infty} f^{\prime}(x)(t-x) H_{n}(t-x) d t d x\right| \\
& +\left|\int_{a}^{\infty} \int_{b}^{\infty} \int_{x}^{t}(t-u) d f^{\prime}(u) H_{n}(t-x) d t d x\right| \\
= & I_{2,1}^{\prime}+I_{2,2}^{\prime} .
\end{aligned}
$$

Now, we see that

$$
\begin{aligned}
I_{2,1}^{\prime} & =\left|\int_{a}^{\infty} \int_{b}^{\infty} f^{\prime}(x)(t-x) H_{n}(t-x) d t d x\right| \\
& =\left|\int_{a}^{\infty} f^{\prime}(x) \int_{x-b}^{\infty} u H_{n}(u) d u d x\right| \\
& =\left|\int_{a-b}^{\infty} u H_{n}(u) \int_{a}^{u+b} f^{\prime}(x) d x d u\right| \\
& \leq \sup _{a \leq x<\infty}\left|f^{\prime}(x)\right|\left|\int_{\delta}^{\infty}(u-\delta) u H_{n}(u) d u\right| \\
& \leq \sup _{[b, \infty)}\left|f^{\prime}(x)\right| O\left(\mu_{n}\right)
\end{aligned}
$$


by (1.3). We estimate $I_{2,2}^{\prime}$. We fix an arbitrary $\eta>0$. Then we have by means of the substitution $u=y+x$ with a new variable $y$

$$
\begin{aligned}
I_{2,2}^{\prime} & =\left|\int_{a}^{\infty} \int_{b}^{\infty} \int_{x}^{t}(t-u) d f^{\prime}(u) H_{n}(t-x) d t d x\right| \\
& =\left|\int_{a}^{\infty} \int_{b}^{\infty} \int_{0}^{t-x}(t-x-y) d f^{\prime}(x+y) H_{n}(t-x) d t d x\right| \\
& \leq 2 \int_{a}^{\infty} \int_{b}^{\infty} \int_{0}^{|t-x|}|t-x|\left|d f^{\prime}(x+y)\right| H_{n}(t-x) d t d x \quad \because|t-x-y| \leq 2|t-x| \\
& \leq 2 \int_{a}^{\infty} \sum_{j=0}^{\infty} \int_{j \eta \leq|t-x| \leq(j+1) \eta} \int_{0}^{(j+1) \eta}\left|d f^{\prime}(x+y)\right||t-x| H_{n}(t-x) d t d x \\
& \leq 2 \sum_{j=0}^{\infty} \int_{j \eta \leq|u| \leq(j+1) \eta}|u| H_{n}(u) d u \int_{a}^{\infty} \int_{0}^{(j+1) \eta}\left|d f^{\prime}(x+y)\right| d x .
\end{aligned}
$$

Then

$$
\begin{aligned}
& \int_{a}^{\infty} \int_{0}^{(j+1) \eta}\left|d f^{\prime}(x+y)\right| d x \\
& \quad=\int_{a}^{\infty} \int_{x}^{x+(j+1) \eta}\left|d f^{\prime}(v)\right| d x \\
& \quad=\int_{a \leq v \leq a+(j+1) \eta} \int_{a \leq x \leq v} d x\left|d f^{\prime}(v)\right|+\int_{a+(j+1) \eta \leq v<\infty} \int_{\nu-(j+1) \eta \leq x \leq v} d x\left|d f^{\prime}(v)\right| \\
& :=A_{1}+A_{2} .
\end{aligned}
$$

Since we can see

$$
A_{1}=\int_{a \leq v \leq a+(j+1) \eta} \int_{a \leq x \leq v} d x\left|d f^{\prime}(v)\right|=\int_{a \leq v \leq a+(j+1) \eta}(v-a)\left|d f^{\prime}(v)\right| \leq(j+1) \eta \operatorname{BV}\left(f^{\prime} ; \mathbb{R}^{+}\right)
$$

and

$$
A_{2}=\int_{a+(j+1) \eta \leq v<\infty} \int_{v-(j+1) \eta \leq x \leq v} d x\left|d f^{\prime}(v)\right| \leq(j+1) \eta \mathrm{BV}\left(f^{\prime} ; \mathbb{R}^{+}\right),
$$

we have

$$
\int_{a}^{\infty} \int_{0}^{(j+1) \eta}\left|d f^{\prime}(x+y)\right| d x \leq 2(j+1) \eta \mathrm{BV}\left(f^{\prime} ; \mathbb{R}^{+}\right)
$$

Now, we estimate $\int_{j \eta \leq|u| \leq(j+1) \eta}|u| H_{n}(u) d u$ for a non-negative integer $j$. Let $j=0$. Then from (1.2) and (1.3), we have

$$
\int_{0 \leq|u| \leq \eta}|u| H_{n}(u) d u \leq\left(\int_{0 \leq|u| \leq \eta} H_{n}(u) d u\right)^{1 / 2}\left(\int_{0 \leq|u| \leq \eta} u^{2} H_{n}(u) d u\right)^{1 / 2}=O\left(\mu_{n}^{1 / 2}\right) .
$$

Let $j \geq 1$. Then by (2.1) we have

$$
\int_{j \eta \leq|u| \leq(j+1) \eta}|u| H_{n}(u) d u \leq C \frac{\mu_{n}^{\beta}}{(j \eta)^{\alpha-1}} .
$$


Therefore, we have

$$
\begin{aligned}
I_{2,2}^{\prime} \leq & \int_{0 \leq|u| \leq \eta}|u| H_{n}(u) d u \int_{a}^{\infty} \int_{0}^{\eta}\left|d f^{\prime}(x+y)\right| d x \\
& +\sum_{j=1}^{\infty} \int_{j \eta \leq|u| \leq(j+1) \eta}|u| H_{n}(u) d u \int_{a}^{\infty} \int_{0}^{(j+1) \eta}\left|d f^{\prime}(x+y)\right| d x \\
\leq & O\left(\mu_{n}^{1 / 2}\right) \eta \mathrm{BV}\left(f^{\prime} ; \mathbb{R}^{+}\right)+\sum_{j=1}^{\infty} \frac{1}{(j \eta)^{\alpha-1}} O\left(\mu_{n}^{\beta}\right)(j+1) \eta \mathrm{BV}\left(f^{\prime} ; \mathbb{R}^{+}\right) \\
= & O\left(\mu_{n}^{1 / 2}\right) \eta \mathrm{BV}\left(f^{\prime} ; \mathbb{R}^{+}\right)+O\left(\mu_{n}^{\beta}\right) \frac{1}{\eta^{\alpha-2}} \mathrm{BV}\left(f^{\prime} ; \mathbb{R}^{+}\right) \quad(\because \alpha>3) .
\end{aligned}
$$

If we let $\eta=\mu_{n}^{1 / 2}$, then we have $I_{2,2}^{\prime}=O\left(\mu_{n}\right) \mathrm{BV}\left(f^{\prime} ; \mathbb{R}^{+}\right)$, because $\beta-(\alpha-2) / 2 \geq 1$. Consequently, (ii) is proved.

(iii) It follows from (2.4). Consequently, for a linear function $f$ on $[b, \infty)$

$$
\left\|J_{n}(f ; x)-f(x)\right\|_{L_{p}([a, \infty))}=O\left(\mu_{n}^{\beta}\right)=o\left(\mu_{n}\right) .
$$

Proof of Theorem 1.3 (i), (ii) hold as follows: Let $f \in L_{p}\left(\mathbb{R}^{+}\right)$. First, we choose $\psi \in C^{2}\left(\mathbb{R}^{+}\right)$ with $\psi^{\prime \prime} \in L_{q}([a, \infty))$ (for any $\left.1 \leq q \leq \infty\right)$ such that

$$
\psi(a)=\psi^{\prime}(a)=\psi^{\prime \prime}(a)=0, \quad \lim _{r \rightarrow \infty} \psi^{(i)}(r)=0, \quad i=0,1,2
$$

and

$$
\psi(x)=0 \quad \text { for } x \notin[a, \infty)
$$

We use the bilinear functional;

$$
A_{n}(f, \psi)=\frac{1}{\mu_{n}} \int_{0}^{\infty}\left(J_{n}(f, x)-f(x)\right) \psi(x) d x
$$

We will show that for fixed $\psi, A_{n}(\cdot, \psi)$ is uniformly bounded on $L_{p}\left(\mathbb{R}^{+}\right)$. We see

$$
\int_{0}^{\infty} J_{n}(f, x) \psi(x) d x=\int_{0}^{\infty} \psi(x) \int_{0}^{\infty} f(t) H_{n}(t-x) d t d x
$$

For $x, t \in[0, \infty)$ we can write

$$
\psi(x)=\psi(t)+\psi^{\prime}(t)(x-t)+\int_{x}^{t}(x-u) \psi^{\prime \prime}(u) d u
$$

Hence,

$$
\begin{aligned}
& \int_{0}^{\infty} \int_{0}^{\infty} f(t) \psi(x) H_{n}(t-x) d x d t \\
& \quad=\int_{0}^{\infty} f(t) \psi(t) \int_{0}^{\infty} H_{n}(t-x) d x d t
\end{aligned}
$$




$$
\begin{aligned}
& +\int_{0}^{\infty} f(t) \psi^{\prime}(t) \int_{0}^{\infty}(x-t) H_{n}(t-x) d x d t \\
& +\int_{0}^{\infty} f(t) \int_{0}^{\infty} \int_{x}^{t}(x-u) \psi^{\prime \prime}(u) d u H_{n}(t-x) d x d t \\
= & I_{1}^{\prime \prime}+I_{2}^{\prime \prime}+I_{3}^{\prime \prime} .
\end{aligned}
$$

From (1.2)

$$
I_{1}^{\prime \prime}=\int_{0}^{\infty} f(t) \psi(t) \int_{-\infty}^{t} H_{n}(u) d u d t=\int_{0}^{\infty} f(t) \psi(t)\left(1-\int_{t}^{\infty} H_{n}(u) d u\right) d t
$$

Since $\psi(t)=0$ for $t \notin[a, \infty)$ (so, we may take $t \geq a$ ), by (2.1) we have

$$
\begin{aligned}
& \left|\int_{0}^{\infty} f(t) \psi(t) \int_{t}^{\infty} H_{n}(u) d u d t\right| \\
& \quad \leq\left|\int_{0}^{\infty} f(t) \psi(t) \int_{t}^{\infty}\left(\frac{u}{t}\right)^{\alpha} H_{n}(u) d u d t\right| \quad \because(u / t)^{\alpha} \geq 1 \\
& \quad \leq O\left(\mu_{n}^{\beta}\right) \int_{a}^{\infty}|f(t) \psi(t)| \frac{1}{t^{\alpha}} d t \quad \text { by }(1.4) \\
& \quad \leq O\left(\mu_{n}^{\beta}\right) \sup _{a \leq t<\infty}|\psi(t)| \int_{a}^{\infty}|f(t)| \frac{1}{t^{\alpha}} d t \\
& \quad \leq O\left(\mu_{n}^{\beta}\right) \sup _{a \leq t<\infty}|\psi(t)|\|f\|_{L_{p}([a, \infty))}\left\|t^{-\alpha}\right\|_{L_{q}([a, \infty))},
\end{aligned}
$$

where $1 / p+1 / q=1$. Thus, we have

$$
I_{1}^{\prime \prime}=\int_{0}^{\infty} f(t) \psi(t) d t+O\left(\mu_{n}^{\beta}\right)\|f\|_{L_{p}([a, \infty))} .
$$

We estimate $I_{2}^{\prime \prime}$. We may $a \leq t$, because $\psi(t)=0$ for $t \notin[a, \infty)$. Noting (1.4) and $\alpha<3$,

$$
\begin{aligned}
\left|I_{2}^{\prime \prime}\right| & =\int_{a}^{\infty}\left|f(t) \psi^{\prime}(t)\right| \int_{t}^{\infty} u H_{n}(u) d u d t \\
& \leq \int_{a}^{\infty}\left|f(t) \psi^{\prime}(t)\right| \int_{t}^{\infty} \frac{u^{\alpha}}{t^{\alpha-1}} H_{n}(u) d u d t \\
& =O\left(\mu_{n}^{\beta}\right) \int_{a}^{\infty}\left|f(t) \psi^{\prime}(t)\right| \frac{1}{t^{\alpha-1}} d t \\
& =O\left(\mu_{n}^{\beta}\right)\left\|\psi^{\prime}\right\|_{L_{\infty}([a, \infty))}\|f\|_{L_{p}([a, \infty))}\left\|t^{1-\alpha}\right\|_{L_{q}([a, \infty))} \\
& =O\left(\mu_{n}^{\beta}\right)\|f\|_{L_{p}([a, \infty))} .
\end{aligned}
$$

Finally, we estimate $I_{3}^{\prime \prime}$. Let $1<p<\infty$ and $1 / p+1 / q=1$. As the estimation for $I_{2,2}$, we have by (1.3)

$$
\begin{aligned}
\left|I_{3}^{\prime \prime}\right| & =\left|\int_{0}^{\infty} f(t) \int_{0}^{\infty} \int_{x}^{t}(x-u) \psi^{\prime \prime}(u) d u H_{n}(t-x) d x d t\right| \\
& =\left|\int_{0}^{\infty} f(t) \int_{0}^{\infty} \frac{1}{t-x} \int_{x}^{t} \psi^{\prime \prime}(u) d u(t-x)^{2} H_{n}(t-x) d x d t\right|
\end{aligned}
$$




$$
\begin{aligned}
& \leq \int_{a}^{\infty}|f(t)| \theta\left(\psi^{\prime \prime}, t\right) \int_{0}^{\infty}(t-x)^{2} H_{n}(t-x) d x d t \\
& =O\left(\mu_{n}\right) \int_{a}^{\infty}|f(t)| \theta\left(\psi^{\prime \prime}, t\right) d t \leq O\left(\mu_{n}\right)\|f\|_{L_{p}([a, \infty))}\left\|\psi^{\prime \prime}\right\|_{L_{q}([a, \infty))} \\
& =O\left(\mu_{n}\right)\|f\|_{L_{p}([a, \infty))} .
\end{aligned}
$$

We estimate $I_{3}^{\prime \prime}$ for $p=1$. There exists $\eta$ between $x$ and $t$ such that

$$
\begin{aligned}
\left|I_{3}^{\prime \prime}\right| & \leq\left|\int_{0}^{\infty} f(t) \int_{0}^{\infty} \psi^{\prime \prime}(\eta)(t-x)^{2} H_{n}(t-x) d x d t\right| \\
& \leq \sup _{a \leq t<\infty}\left|\psi^{\prime \prime}(t)\right| \int_{a}^{\infty}|f(t)| \int_{0}^{\infty}(t-x)^{2} H_{n}(t-x) d x d t \\
& \leq O\left(\mu_{n}\right) \sup _{a \leq t<\infty}\left|\psi^{\prime \prime}(t)\right|\|f\|_{L_{1}([a, \infty))}=O\left(\mu_{n}\right)\|f\|_{L_{1}([a, \infty)) .}
\end{aligned}
$$

Here we used (1.3). Consequently, it follows that $\left|A_{n}(f, \psi)\right|$ is uniformly bounded on $L_{p}\left(\mathbb{R}^{+}\right)$. Next, from Theorem 1.1 we see that for $f \in C_{c}^{2}\left(\mathbb{R}^{+}\right)$,

$$
\lim _{n \rightarrow \infty} A_{n}(f, \psi)=\frac{1}{2} \int_{a}^{\infty} f^{\prime \prime}(x) \psi(x) d x=\frac{1}{2} \int_{a}^{\infty} f(x) \psi^{\prime \prime}(x) d x .
$$

Since $\left\{A_{n}(\cdot, \psi)\right\}$ is uniformly bounded on $L_{p}\left(\mathbb{R}^{+}\right)$, and $C_{c}^{2}\left(\mathbb{R}^{+}\right)$is dense in $L_{p}\left(\mathbb{R}^{+}\right)$, (3.14) yields

$$
\lim _{n \rightarrow \infty} A_{n}(f, \psi)=\frac{1}{2} \int_{a}^{\infty} f(x) \psi^{\prime \prime}(x) d x
$$

for any $f \in L_{p}\left(\mathbb{R}^{+}\right)$. Now, for any fixed $f \in L_{p}\left(\mathbb{R}^{+}\right)$, we consider the sequence of linear functional $\left\{A_{n}(f, \cdot)\right\}$. Since $\left\|J_{n}(f)-f\right\|_{L_{p}([a, \infty))}=O\left(\mu_{n}\right), n \rightarrow \infty$, there exist $h \in L_{p}([a, \infty))(p>1)$ and $h \in \mathrm{BV}[a, \infty)(p=1)$ and a subsequence $\left\{A_{n_{j}}(f, \cdot)\right\}$ such that

$$
\lim _{j \rightarrow \infty} A_{n_{j}}(f, \psi)= \begin{cases}\int_{0}^{\infty} h(x) \psi(x) d x, & p>1, \\ \int_{0}^{\infty} \psi(x) d h(x), & p=1 .\end{cases}
$$

From (3.15) and (3.16) we obtain

$$
\frac{1}{2} \int_{0}^{\infty} f(x) \psi^{\prime \prime}(x) d x= \begin{cases}\int_{0}^{\infty} h(x) \psi(x) d x, & p>1 \\ \int_{0}^{\infty} \psi(x) d h(x), & p=1 .\end{cases}
$$

A particular solution to (3.17) is

$$
\frac{1}{2} f(x)= \begin{cases}\int_{\eta}^{x} \int_{\eta}^{\xi} h(\mu) d \mu d \xi, & p>1 \\ \int_{\eta}^{x} \int_{\eta}^{\xi} d h(\mu) d \xi, & p=1\end{cases}
$$

The homogeneous problem

$$
\int_{a}^{\infty} f(x) \psi^{\prime \prime}(x) d x=0
$$


has the general solution $f(x)=C_{1} x+C_{2}$ for $a \leq x<\infty$, since we can take $\psi \in C^{2}([a, \infty))$ arbitrarily as $\psi^{\prime \prime} \in L_{q}([a, \infty)), \psi(a)=\psi^{\prime}(a)=0, \lim _{r \rightarrow \infty} \psi^{(i)}(r)=0, i=0,1$. Hence, if $1<$ $p<\infty, f \in L_{p}^{2}([a, \infty))$, and if $p=1$ then $f^{\prime} \in \mathrm{BV}([a, \infty))$. Hence, (i) and (ii) hold. We will show (iii). Now, if

$$
\left\|J_{n}(f)-f\right\|_{L_{p}([a, \infty))}=o\left(\mu_{n}\right), \quad n \rightarrow \infty,
$$

then

$$
\begin{aligned}
\left|A_{n}(f, \psi)\right| & \leq \frac{1}{\mu_{n}} \int_{a}^{\infty}\left|J_{n}(f, x)-f(x) \| \psi(x)\right| d x \\
& \leq\left(\sup _{a \leq x<\infty}|\psi(x)|\right) \frac{C_{p}}{\mu_{n}}\left\|J_{n}(f)-f\right\|_{L_{p}([a, \infty))},
\end{aligned}
$$

where $C_{p}>0$ is independent of $n$. Hence

$$
\lim _{n \rightarrow \infty} A_{n}(f, \psi)=0
$$

Considering (3.15), (3.16), (3.17), and (3.18), we obtain

$$
\int_{a}^{\infty} f(x) \psi^{\prime \prime}(x) d x=0
$$

and so

$$
\int_{a}^{\infty} f^{\prime \prime}(x) \psi(x) d x=0
$$

consequently, we have $f^{\prime \prime}(x)=0$, that is, $f$ is linear on $[a, \infty)$.

\section{Competing interests}

The authors declare that they have no competing interests.

\section{Authors' contributions}

All authors conceived of the study, participated in its design and coordination, drafted the manuscript and participated in the sequence alignment. All authors read and approved the final manuscript.

\section{Author details}

${ }^{1}$ Department of Mathematics Education, Sungkyunkwan University, Seoul, 110-745, Republic of Korea. ${ }^{2}$ Department of Mathematics, Meijo University, Nagoya, 468-8502, Japan.

\section{Acknowledgements}

The authors thank the referees for many valuable comments and corrections.

Received: 27 December 2013 Accepted: 22 August 2014 Published: 02 Sep 2014

\section{References}

1. Swetits, JJ, Wood, B: Local $L_{p}$-saturation of positive linear convolution operators. J. Approx. Theory 34(4), 348-360 (1982)

2. Swetits, JJ, Wood, B: Approximating functions of exponential type on the positive axis: direct results. Bull. Inst. Math. Acad. Sin. 23(2), 167-180 (1995)

3. Jung, HS, Sakai, R: Approximation by Lupas-type operators and Szász-Mirakyan-type operators. J. Appl. Math. 2012 Article ID 546784 (2012)

4. Deo, N, Jung, HS, Sakai, R: Degree of approximation by hybrid operators. Abstr. Appl. Anal. 2013, Article ID 732069 (2013)

5. Mamedov, RG: The asymptotic value of the approximation of differentiable functions by linear positive operators. Dokl. Akad. Nauk SSSR 128, 471-474 (1959) (Russian)

6. Stein, EM: Harmonic Analysis: Real-Variable Methods, Orthogonality, and Oscillatory Integrals. Princeton University Press, Princeton (1993) 
10.1186/1029-242X-2014-329

Cite this article as: Jung and Sakai: Local saturation of a positive linear convolution operator. Journal of Inequalities and Applications 2014, 2014:329

Submit your manuscript to a SpringerOpen ${ }^{\circ}$ journal and benefit from:

- Convenient online submission

- Rigorous peer review

- Immediate publication on acceptance

Open access: articles freely available online

- High visibility within the field

- Retaining the copyright to your article

Submit your next manuscript at $\boldsymbol{s p r i n g e r o p e n . c o m ~}$ 